\title{
Influence of Pressure on the Electron-Phonon Interaction in Superconductors
}

\author{
Mat. OrendáC̆ $\check{C}^{a, b, *}$, S. GabÁni ${ }^{a}$, G. Pristás̆ ${ }^{a}$, E. GaŽO $^{a}$, N. Shitsevalova ${ }^{c}$ \\ AND K. FLACHBART ${ }^{a}$ \\ ${ }^{a}$ Institute of Experimental Physics, SAS, Watsonova 47, 04001 Košice, Slovakia \\ ${ }^{b}$ Institute of Physics, Faculty of Sciences, P.J. Safarik University, Park Angelinum 9, 04001 Košice, Slovakia \\ ${ }^{c}$ Institute for Problems of Materials Science, NASU, Krzyzhanovsky 3, 03680 Kiev, Ukraine
}

\begin{abstract}
The electron-phonon interaction is a very important and ubiquitous process in solids, affecting almost all their physical properties. In metals, where the relaxation processes depend on both electrons and phonons, all thermodynamic and transport properties are dictated by the electron-phonon interaction. A very strong manifestation of the electron-phonon interaction is related with the superconducting state. Here we report the effect of high pressure on the transport electron-phonon interaction, $\lambda_{t r}$, in superconducting systems such as $\mathrm{YB}_{6}\left(T_{c} \approx 7.5 \mathrm{~K}\right), \mathrm{Pb}$ $\left(T_{c} \approx 7.2 \mathrm{~K}\right), \mathrm{Nb}$ bulk or thin film $\left(T_{c} \approx 9.2 \mathrm{~K}\right)$, and in $\mathrm{LaB}_{6}$ in which superconductivity was not yet observed. The expected pressure effect should correspond to the theoretically predicted negative pressure effect on $T_{c}$ (except for $\mathrm{Nb}$ thin film and $\mathrm{LaB}_{6}$ ). To determine the influence of pressure on $\lambda_{t r}$, we utilized the Bloch-Grüneisen fit (denoted also as "thermal spectroscopy") of the precise temperature dependence of resistivity measurements in the normal state up to a pressure of $2.8 \mathrm{GPa}$. Based on this fit the observed negative pressure effect on $\lambda_{t r}$ values, $\mathrm{d} \lambda_{t r} / \mathrm{d} p$, are as follows: $\mathrm{d} \lambda_{t r} / \mathrm{d} p \approx-0.045 \mathrm{GPa}^{-1}$ for $\mathrm{YB}_{6}, \mathrm{~d} \lambda_{t r} / \mathrm{d} p \approx-0.13 \mathrm{GPa}^{-1}$ for $\mathrm{Pb}, \mathrm{d} \lambda_{t r} / \mathrm{d} p \approx-0.019 \mathrm{GPa}^{-1}$ or $-0.028 \mathrm{GPa}^{-1}$ for Nb bulk or thin film, respectively, and $\mathrm{d} \lambda_{t r} / \mathrm{d} p \approx-0.003 \mathrm{GPa}^{-1}$ for $\mathrm{LaB}_{6}$.
\end{abstract}

DOI: 10.12693/APhysPolA.131.1039

PACS/topics: 74.70.Ad, 72.15.Eb, 74.62.Fj, 74.25.F-, 63.20.kd

\section{Introduction}

The electron-phonon interaction (EPI) exists in solids due to scattering of electrons on vibrating lattice ions (phonons). It affects their transport and thermodynamic properties, and is very significantly manifested in superconducting materials $[1,2]$. EPI is characterized by the coupling parameter $\lambda$ and it can be measured or calculated by various methods. Empirical $\lambda$ values of some superconducting elements and compounds at ambient pressure received from transport $\left(\lambda_{t r}\right)$ or spectroscopic measurements can be found e.g. in [1-6]. Experimental [7] or theoretical $[1,8,9]$ studies of the pressure effect on EPI, however, are very rare and each new result is welcomed.

As to the materials, $\mathrm{Pb}\left(T_{c} \approx 7.2 \mathrm{~K}, \lambda_{t r} \approx 1.48\right)$ is a BCS type-1 superconductor which crystallizes in fcc lattice. Due to its pronounced and smooth pressure effect on $T_{c}\left(\mathrm{~d} T_{c} / \mathrm{d} p=-0.360 \mathrm{~K} / \mathrm{GPa}\right)$ it is used as a manometer in high pressure cells. $\mathrm{Nb}$ is a type- 2 superconductor $\left(T_{c} \approx 9.2 \mathrm{~K}, \lambda_{t r} \approx 1.06\right)$, which crystallizes in bcc lattice. $\mathrm{YB}_{6}$ and $\mathrm{LaB}_{6}$ crystallize also in bcc structure, and $\mathrm{LaB}_{6}$ $(\lambda \approx 0.19)$ is an isostructural system to superconducting $\mathrm{YB}_{6}\left(T_{c} \approx 7.5 \mathrm{~K}, \lambda_{t r} \approx 1.03\right)$.

Our investigation of the EPI under pressure was based on the method developed for $\mathrm{YB}_{6}$ in [10], in which the normal state resistivity measurement, $\rho(T)$, as "thermal spectroscopy" was deconvolved into a spectrum of the EPI and the coupling constant $\lambda_{t r}$. Such an approach was

*corresponding author; e-mail: orendacm@saske.sk successful because $\mathrm{YB}_{6}$ happened to be an example of a superconductor with a dominant low-energy Einstein-like phonon mode $(\approx 8 \mathrm{meV})$.

\section{Thermal spectroscopy}

The temperature dependence of electrical resistivity of simple superconductors in normal state can be described by the Bloch-Grüneisen (BG) formula [10]:

$$
\rho_{B G}(T)=\rho_{0}+\frac{4 \pi m_{b}}{n e^{2}} \int_{0}^{\omega_{\max }} \alpha_{t r}^{2} F(\omega) \frac{x \mathrm{e}^{x}}{\left(\mathrm{e}^{x}-1\right)^{2}} \mathrm{~d} \omega,(1)
$$

where $x \equiv \omega / T, T$ is temperature, $\omega$ - the frequency of phonons, $m_{b}$ - the electron effective mass near the Fermi level, $n$ - the electron concentration, $e$ - the electron charge, $\alpha_{t r}^{2} F(\omega)$ - the Eliashberg spectral function, $\omega_{\max }$ - the maximum frequency in the phonon density of states (PDOS) and $\rho_{0}$ the temperature independent residual resistivity.

In materials with significant peaks in PDOS it is possible to approximate the PDOS by its decomposition into a set of the Einstein phonon modes [10]:

$$
\alpha_{t r}^{2} F(\omega)=\frac{1}{2} \sum_{k} \lambda_{t r, k} \omega_{k} \delta\left(\omega-\omega_{k}\right),
$$

where $\lambda_{t r, k}$ is the electron-phonon transport coupling parameter for the $k$-th phonon mode and $\omega_{k}$ is its frequency. By substituting (2) into (1) one obtains the discrete version of BG formula [10]:

$$
\rho_{B G}^{\prime}(T)=\rho_{0}+\frac{2 \pi}{\varepsilon_{0} \Omega_{p}^{2}} \sum_{k} \lambda_{t r} \omega_{k} \frac{x_{k} \mathrm{e}^{x_{k}}}{\left(\mathrm{e}^{x_{k}}-1\right)^{2}}
$$

where $x_{k} \equiv \omega_{k} / T, \Omega_{p}^{2}=\left(n e^{2} / \varepsilon_{0} m_{b}\right)$ is the unscreened plasma frequency and $\varepsilon_{0}$ - the permittivity of vacuum. 
By taking into account the negative curvature of resistivity at high temperature, which is possibly related to the Mott limit $\rho_{\max }[10]$, one can obtain the fitting $\rho(T)$ dependence using the empirical "parallel resistor" formula

$$
\frac{1}{\rho(T)}=\frac{1}{\rho_{B G}^{\prime}(T)}+\frac{1}{\rho_{\max }} .
$$

In our case the thermal spectroscopy method consisted of measuring the temperature dependence of resistivity at various pressures and fitting of the measured data by (4). From corresponding fit, values of the EPI parameter $\lambda_{t r, k}$ and the frequencies of Einstein modes $\omega_{k}$ were obtained.

\section{Experimental details}

Electrical resistance was measured by a standard fourwire method with lock-in amplifier. For pressure generation, a $\mathrm{CuBe}$ piston cylinder pressure cell with $\mathrm{NiCrAl}$ core and dielectric oil DAPHNE as pressure medium were used. Pressure was indicated from the superconducting transition of lead.

\section{Results and discussion}

In the temperature derivation of $\rho(T)$ of $\mathrm{Pb}$ (Fig. 1) there was observed a pronounced maximum, which is shifting with increase of pressure to higher temperatures. In thermal spectroscopy model this behaviour is observed when the Einstein-like phonon mode increases its frequency. This indicates the hardening of the Einstein-like phonon mode, which is responsible for the superconducting coupling. Electron-phonon transport coupling parameter $\lambda_{t r, 1}$ for softer phonon mode in $\mathrm{Pb}$ (Fig. 2) has larger value than $\lambda_{t r, 2}$ for higher frequency one.

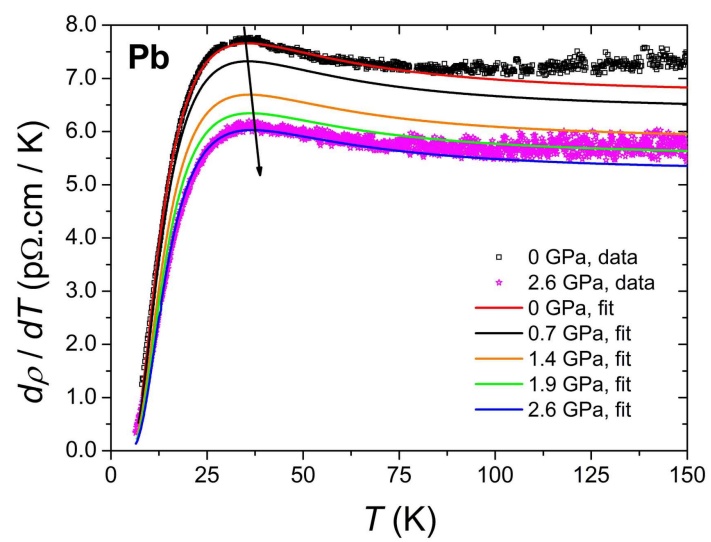

Fig. 1. Temperature dependences of $\mathrm{d} \rho(T) / \mathrm{d} T$ for $\mathrm{Pb}$ together with BG fits at various pressures. Inset shows $\rho(T) / \rho(0)$ dependences (for clarity not all data points are shown) at ambient and maximum pressures with fits.

Neutron spectroscopy investigations of $\mathrm{Pb}$ show two significant peaks in PDOS at $\approx 4.3 \mathrm{meV}$ and $\approx 9 \mathrm{meV}[1]$, which are responsible for its EPI and superconductivity. In our case $\rho(T)$ at ambient pressure was at first fitted by the two-term-discrete BG formula (3) using as starting parameters for the Einstein frequencies of phonon modes $\hbar \omega_{E, 1}=4.3 \mathrm{meV}$ and $\hbar \omega_{E, 2}=9 \mathrm{meV}$. From performed fit the parameters $\lambda_{t r, 1}$ and $\lambda_{t r, 2}$ for each phonon mode were obtained (Fig. 2).

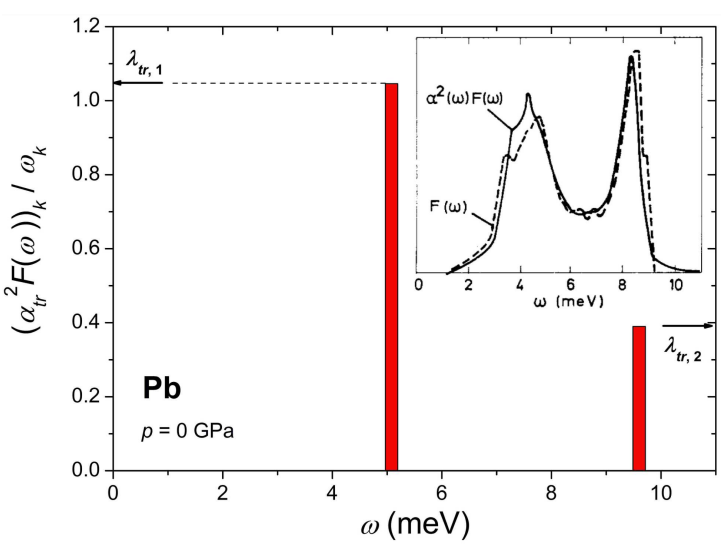

Fig. 2. Transport electron-phonon coupling parameters, $\lambda_{t r, 1}$ and $\lambda_{t r, 2}$, of $\mathrm{Pb}$ deconvolved from the temperature dependence of resistivity at ambient pressure. In the inset, the phonon density of states, $F(\omega)$, and the Eliashberg spectral function, $\alpha^{2} F(\omega)$, of $\mathrm{Pb}$ from neutron spectroscopy investigation are shown [1].

The same fitting procedure was used for all applied pressures and from them the total parameters, $\lambda_{t r}=$ $\lambda_{t r, 1}+\lambda_{t r, 2}$, were obtained. Based on $\lambda_{t r}$ data (Fig. 3) a negative pressure effect on the transport EPI parameter of $\mathrm{Pb}, \mathrm{d} \lambda_{t r} / \mathrm{d} p \approx-0.13 \mathrm{GPa}^{-1}$, was observed. This value is in good agreement with the theoretical value, $\mathrm{d} \lambda_{t r} / \mathrm{d} p \approx-0.17 \mathrm{GPa}^{-1}$, received from theoretical prediction in [9], where a multiple-cutoff Lorentzian model of PDOS was used.

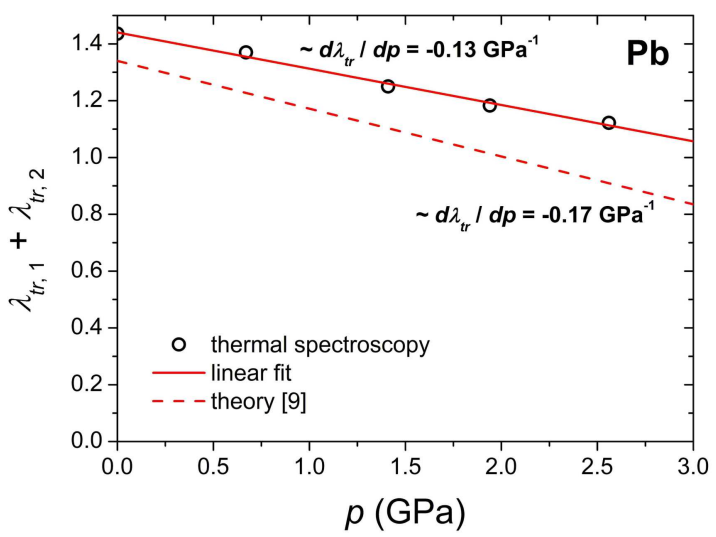

Fig. 3. Pressure dependence of the transport EPI parameter, $\lambda_{t r}$, of $\mathrm{Pb}$ obtained from thermal spectroscopy (circles) and given by theoretical calculation from [9] (dashed line).

The same procedure was used for other studied samples, $\mathrm{YB}_{6}, \mathrm{LaB}_{6}, \mathrm{Nb}$ bulk and $\mathrm{Nb}$ thin film (100 nm), respectively. From pressure dependences of the difference between critical temperature at applied and ambient pressure (Fig. 4) one can see that $T_{c}$ of $\mathrm{YB}_{6}, \mathrm{~Pb}$ and of bulk $\mathrm{Nb}$ decreases with pressure linearly up to $2.8 \mathrm{GPa}$. 
On the other hand, for the $\mathrm{Nb}$ thin film $T_{c}(p)$ increases with pressure. For $\mathrm{LaB}_{6}$, no superconducting transition was observed down to $1.6 \mathrm{~K}$.

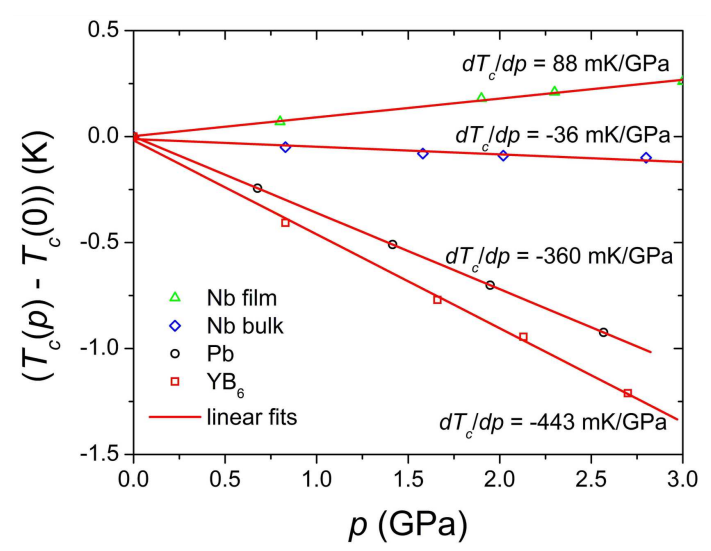

Fig. 4. Pressure dependences of the difference between the critical temperature at applied and at ambient pressure for all studied samples.

From pressure dependences of the difference between transport EPI parameter at applied pressure, $\lambda_{t r}(p)$, and at ambient pressure, $\lambda_{t r}(0)$, shown in Fig. 5 , one can see that it decreases with pressure for all our studied samples as follows: $\mathrm{d} \lambda_{t r} / \mathrm{d} p \approx-0.13 \mathrm{GPa}^{-1}$ for $\mathrm{Pb}, \mathrm{d} \lambda_{t r} / \mathrm{d} p \approx$ $-0.045 \mathrm{GPa}^{-1}$ for $\mathrm{YB}_{6}, \mathrm{~d} \lambda_{t r} / \mathrm{d} p \approx-0.019 \mathrm{GPa}^{-1}$ for Nb bulk, $\mathrm{d} \lambda_{t r} / \mathrm{d} p \approx-0.028 \mathrm{GPa}^{-1}$ for $\mathrm{Nb}$ thin film, and $\mathrm{d} \lambda_{t r} / \mathrm{d} p \approx-0.003 \mathrm{GPa}^{-1}$ for $\mathrm{LaB}_{6}$. The received value for $\mathrm{Nb}$ agrees with that theoretically calculated using a full potential LMTO (linear muffin-tin orbital) method in [8].

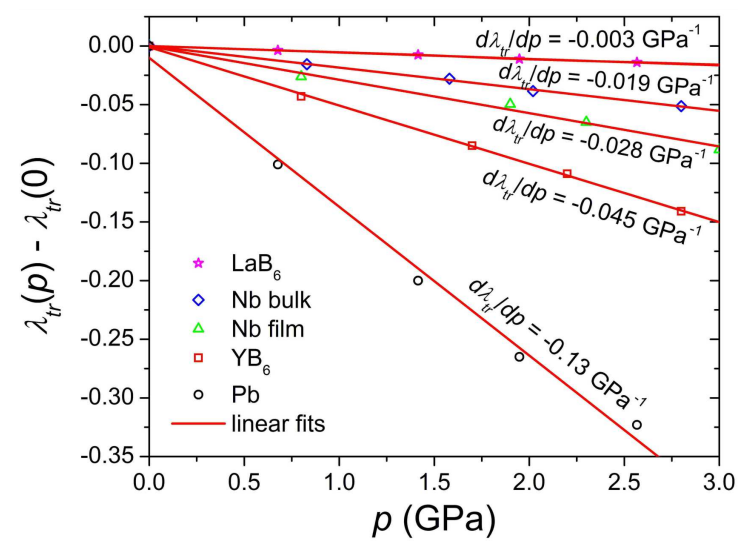

Fig. 5. Pressure dependences of the difference between the transport EPI parameter at applied and at ambient pressure for all studied samples.
As it was shown, for $\mathrm{YB}_{6}, \mathrm{~Pb}, \mathrm{Nb}$ (bulk), both $T_{c}$ and $\lambda_{t r}$ decrease with pressure, as theoretically predicted. Therefore, we can conclude that in these materials mainly the EPI is responsible for their superconducting properties. In $\mathrm{Nb}$ thin film, on the contrary, $T_{c}$ increases with pressure but $\lambda_{t r}$ drops with pressure. Therefore, in Nb thin film some additional mechanism which influences superconductivity, like surface electron-phonon coupling, may be present. In $\mathrm{LaB}_{6}$ no superconducting transition under pressure up to $2.8 \mathrm{GPa}$ was observed and $\lambda_{t r}$ decreases with increasing pressure. From it follows that in this compound superconductivity will likely not be induced by pressure.

\section{Conclusions}

Electrical resistivity based thermal spectroscopy was successfully used to determine the pressure dependence of the EPI parameter in $\mathrm{YB}_{6}, \mathrm{LaB}_{6}, \mathrm{~Pb}, \mathrm{Nb}$ bulk and $\mathrm{Nb}$ thin film up to $2.8 \mathrm{GPa}$. Pressure dependences of $T_{c}$ and $\lambda_{t r}$ indicate that in $\mathrm{YB}_{6}, \mathrm{~Pb}$ and $\mathrm{Nb}$ bulk superconductivity is mediated mainly by bulk phonons, and that in $\mathrm{Nb}$ thin film it might be influenced also by surface phenomena.

\section{Acknowledgments}

This work was supported by project VEGA 2/0032/16, APVV-0605-14 and EU ERDF-ITMS 26220120047.

\section{References}

[1] G. Grimvall, Phys. Scr. 14, 63 (1976).

[2] P.B. Allen, in: Handbook of Superconductivity, Ed. C.P. Poole, Jr., Academic Press, New York 1999.

[3] Yu.S. Ponosov, S.V. Streltsov, Phys. Rev. B 86, 045138 (2012).

[4] Yu.S. Ponosov, A.A. Makhnev, S.V. Streltsov, V.B. Filipov, N.Yu. Shitsevalova, JETP Lett. 102, 503 (2015).

[5] P. Samuely, M. Reiffers, K. Flachbart, A.I. Akimenko, I.K. Yanson, N.M. Ponomarenko, Yu.B. Paderno, J. Low. Temp. Phys. 71, 49 (1988).

[6] I.K. Yanson, Yu.G. Naidyuk, Point-Contact Spectroscopy, Springer-Verlag, New York 2005.

[7] V.V. Struzhkin, Y.A. Timofeev, R.J. Hemley, H. Mao, Phys. Rev. Lett. 79, 4262 (1997).

[8] J.S. Tse, Z. Li, K. Uehara, Y. Ma, Phys. Rev. B 69, 132101 (2004).

[9] R.E. Hodder, Phys. Rev. 180, 530 (1969).

[10] R. Lortz, Y. Wang, U. Tutsch, S. Abe, C. Meingast, P. Popovich, W. Knafo, N. Shitsevalova, Yu.B. Paderno, A. Junod, Phys. Rev. B 73, 024512 (2006). 